\title{
Post-Discharge Symptoms among Hospitalized COVID-19 Patients in Nigeria: A Single-Center Study
}

\author{
Dimie Ogoina, ${ }^{1 *}$ Hendris Izibewule James, ${ }^{1}$ and Stanley Zikeyi Ogoinja ${ }^{2}$ \\ ${ }^{1}$ Department of Internal Medicine, Niger Delta University Teaching Hospital, Okolobiri, Bayelsa State, Nigeria; ${ }^{2}$ Department of Radiology, Niger Delta \\ University Teaching Hospital, Okolobiri, Bayelsa State, Nigeria
}

\begin{abstract}
There is a paucity of studies on post-acute COVID-19 syndrome (PCS) among hospitalized COVID-19 survivors from Nigeria. We describe the frequency, types, and duration of post-discharge symptoms suggestive of PCS among previously hospitalized COVID-19 patients in a treatment center in Nigeria. We conducted a retrospective review of admission and post-discharge follow-up medical records of COVID-19 survivors admitted between April and December 2020. A standardized checklist was used to document post-discharge symptoms. PCS was defined as persisting or new postdischarge symptoms lasting at least 3 weeks after initial COVID-19 symptoms. The relationship between study variables and development of PCS was ascertained by univariate analysis. Thirty of 51 previously hospitalized COVID-19 patients (median age, 46 years; male, 66.7\%) were studied. Seventeen (56.7\%) of the 30 patients developed features suggestive of PCS. Approximately three post-discharge symptoms were reported per patient over a follow-up period of ranging from 3 weeks to 9 months after initial COVID-19 symptoms. Cough, fatigue, and dyspnea were the most common postdischarge symptoms reported. A few patients had symptoms suggestive of thrombosis and COVID-19 reinfection. Among all study variables, baseline COVID-19 severity was the only significant variable associated with the development of PCS. PCS is common in our setting and is characterized by multisystemic signs and symptoms that require vigilance by clinicians for appropriate diagnosis and treatment. Long-term multicenter prospective studies are needed to characterize fully the burden of PCS among COVID-19 survivors in Nigeria.
\end{abstract}

\section{INTRODUCTION}

The COVID-19 pandemic continues to ravage health systems across the globe. The disease is typically characterized by acute symptoms of fever, cough, and shortness of breath, as well as other multisystemic signs and symptoms. Most patients recover completely within 2 weeks of first symptoms, but recovery may take 3 to 6 weeks in severe cases. ${ }^{1}$ However, there is now evidence that COVID-19 symptoms may persist or reoccur in some individuals even after the initial recovery period. ${ }^{2,3}$ These post-recovery symptoms have been called various names, such as post-acute COVID-19 syndrome (PCS), long COVID-19, and post-acute sequalae of severe acute respiratory syndrome 2 (SARS-CoV-2) infection. ${ }^{2-4}$

Amenta et al. ${ }^{3}$ have suggested three categories of PCS, including 1) residual symptoms that persist after recovery from acute infection, 2) organ dysfunction that persists after initial recovery, and 3) new symptoms or syndromes that develop after initial asymptomatic or mild infection. Fatigue, dyspnea, joint pain, and cough have been described as the most common manifestations among patients with PCS, but sequelae have been reported in diverse organ systems. ${ }^{2,3,5}$

As April 29, 2021, Nigeria has reported 165,055 cases, 155,041 recoveries, and 2,063 deaths resulting from COVID19. ${ }^{6}$ Although the spectrum of clinical presentations of acute COVID-19 have been described by various authors from Nige$\mathrm{ria}^{7-9}$ there is a paucity of studies on post-discharge symptoms among hospitalized COVID-19 patients from Nigeria. A study from an outpatient clinic in Lagos state, southwest Nigeria, revealed that $40.6 \%$ of patients had features of PCS after a median follow-up period of about 2 weeks after discharge. ${ }^{10}$ To extend the understanding of post-acute COVID-19 in

*Address correspondence to Dimie Ogoina, Department of Medicine, Niger Delta University/Niger Delta University Teaching Hospital, Bayelsa State, Nigeria, PMB 100 Yenagoa. E-mail: dimieogoina@gmail.com
Nigeria, we describe post-discharge symptoms among hospitalized COVID-19 patients on follow-up at a COVID-19 treatment center in Bayelsa State, south-south Nigeria.

\section{METHODS}

Study site and design. We conducted a retrospective review of admission and follow-up hospital records of a cohort of COVID-19 patients admitted to the Niger Delta University Teaching Hospital COVID-19 treatment center between April and December 2020. The Niger Delta University Teaching Hospital is the main treatment center for COVID-19 in Bayelsa State, south-south Nigeria. The treatment center consists of a 30-bed isolation unit, including a six-bed high-dependency unit for the care of severely ill COVID-19 patients.

Confirmatory diagnosis, admission, treatment, and discharge of all patients were generally in line with Nigeria's COVID-19 treatment and care guidelines. ${ }^{11}$ Specifically, between April and June 2020, the national guidelines required admission of all confirmed cases of COVID-19 regardless of severity of disease. In late June 2020, home-based treatment was introduced for asymptomatic and mild COVID-19 cases, whereas patients with moderate, severe, and critical COVID19 were hospitalized. COVID-19 treatment included supportive care, oxygen replacement therapy, and treatment of comorbidities and complications as necessary.

The discharge criteria during the study period were absence of fever for 3 consecutive days, improvement in other symptoms, and two negative test results for SARS-CoV-2 24 hours apart. ${ }^{11}$ We routinely monitored all discharged patients 1 week post-discharge and then periodically (2- to 4-week intervals) as clinical condition dictated. A standardized symptoms checklist was used to document persistence of symptoms from admission and emergence of new symptoms after discharge.

Study participants and data collection. We included all patients who met the discharge criteria and presented for 
follow-up at least once after discharge. The admission and follow-up medical records of each study participant were reviewed, and sociodemographic data, clinical history, comorbidities, disease severity on admission, and duration of hospital stay were documented in an Microsoft Excel 365 (Microsoft Corp., Redmond, WA) spreadsheet. We also documented dates of follow-up, and the types and number of symptoms at each follow-up visit. PCS was defined as persisting or new COVID-19-related symptoms observed after discharge and at least 3 weeks after initial symptoms of COVID-19. ${ }^{3}$ We excluded any symptom observed during follow-up that was linked causally to other preexisting or new COVID-19 unrelated illnesses.

Data analysis. We used the Statistical Package for Social Sciences (version 20) for data analysis. Qualitative variables were summarized using frequencies and percentages, whereas quantitative variables were summarized using median and interquartile ranges (IQRs). The relationship between the development of PCS and study variables was determined using the Mann-Whitney test, the $\chi^{2}$ test, and Fisher's exact test, as applicable. A $P$ value of $<0.05$ was taken as statistically significant (two-tailed).

Ethical approval. Ethical approval for the study was obtained from the ethical committee of the Niger Delta University Teaching Hospital, Okolobiri, Bayelsa.

\section{RESULTS}

Thirty COVID-19 patients (males, 66.7\%) were eligible for inclusion as study participants out of 51 patients admitted during the study period. Fifteen mortalities and six losses to follow-up were excluded. The age range of study participants was 13 to 63 years, with a median age of 46 years (IQR, 32.5-53 years). The symptoms and signs on admission and at follow-up are shown in Figure 1. Fever, anorexia, headache, and cough were the most common symptoms during admission, whereas cough, fatigue, and shortness of breath were the most common symptoms post-discharge. Seventeen $(56.7 \%)$ of the 30 patients had symptoms suggestive of PCS. The prevalence of PCS in our study was $56.7 \%$. One to five symptoms were reported per patient, with a median of three symptoms (IQR, 2-3.25) per patient.

Duration of follow-up and associated symptoms. The duration from first symptom to last follow-up for each patient ranged from 21 to 267 days, with a median of 39.5 days (IQR, 28.8-75.3 days). The number of days of follow-up after discharge ranged from 7 to 238 days, with a median of 15.5 days (IQR, 7-48.3 days). The number of cases seen at each day of follow-up, and associated symptoms, are shown in Figure 2. Respiratory symptoms such as cough, chest pain, shortness of breath, and throat discomfort were common, but patients also reported fatigue, poor concentration, diarrhea, and anorexia (Figure 2).

Two asymptomatic patients on day 7 of follow-up later developed symptoms on days 14 and 30 . One of these previously asymptomatic patients is a 28-year-old health-care worker who developed fever and dry cough 30 days after a previously negative polymerase chain reaction (PCR) test for COVID-19 and 6 weeks after the first diagnosis of COVID19. A repeat PCR test confirmed COVID-19, but viral culture could not be done, and genomic sequencing of patient samples was not available at the time of this report. The second patient was a 46-year-old housewife who developed chest pain and throat discomfort on day 14 of follow-up.

Lower limb numbness and leg pain were reported by four patients on days 7,21 , and 58 of follow-up. Two of these patients reported associated painful leg swelling and were

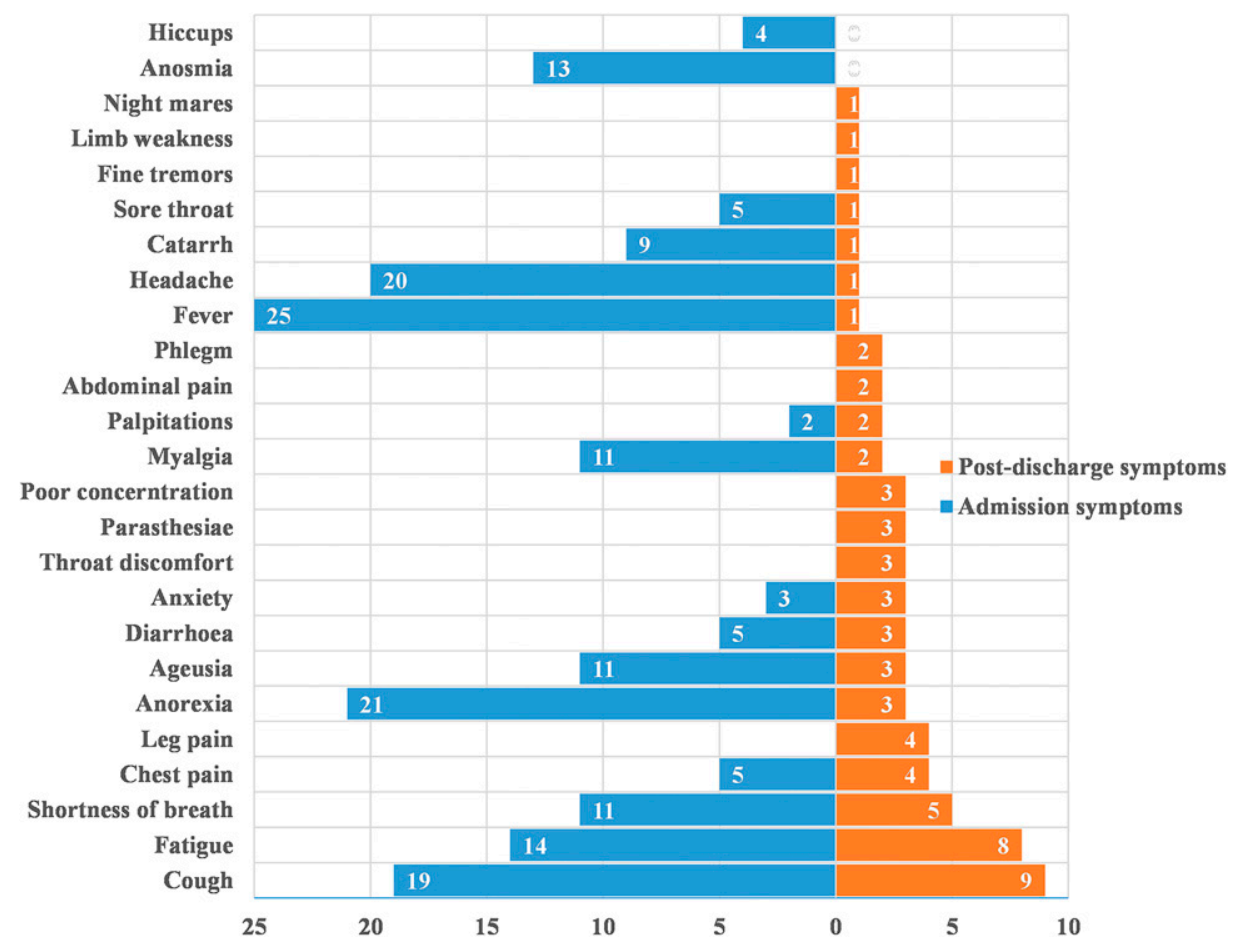

FIGURE 1. Admission and post-discharge symptoms and signs of coronavirus disease 2019 survivors. The most common post-discharge symptoms were cough, fatigue, and shortness of breath. Two of the four patients with leg pain had evidence of deep vein thrombosis. This figure appears in color at www.ajtmh.org. 


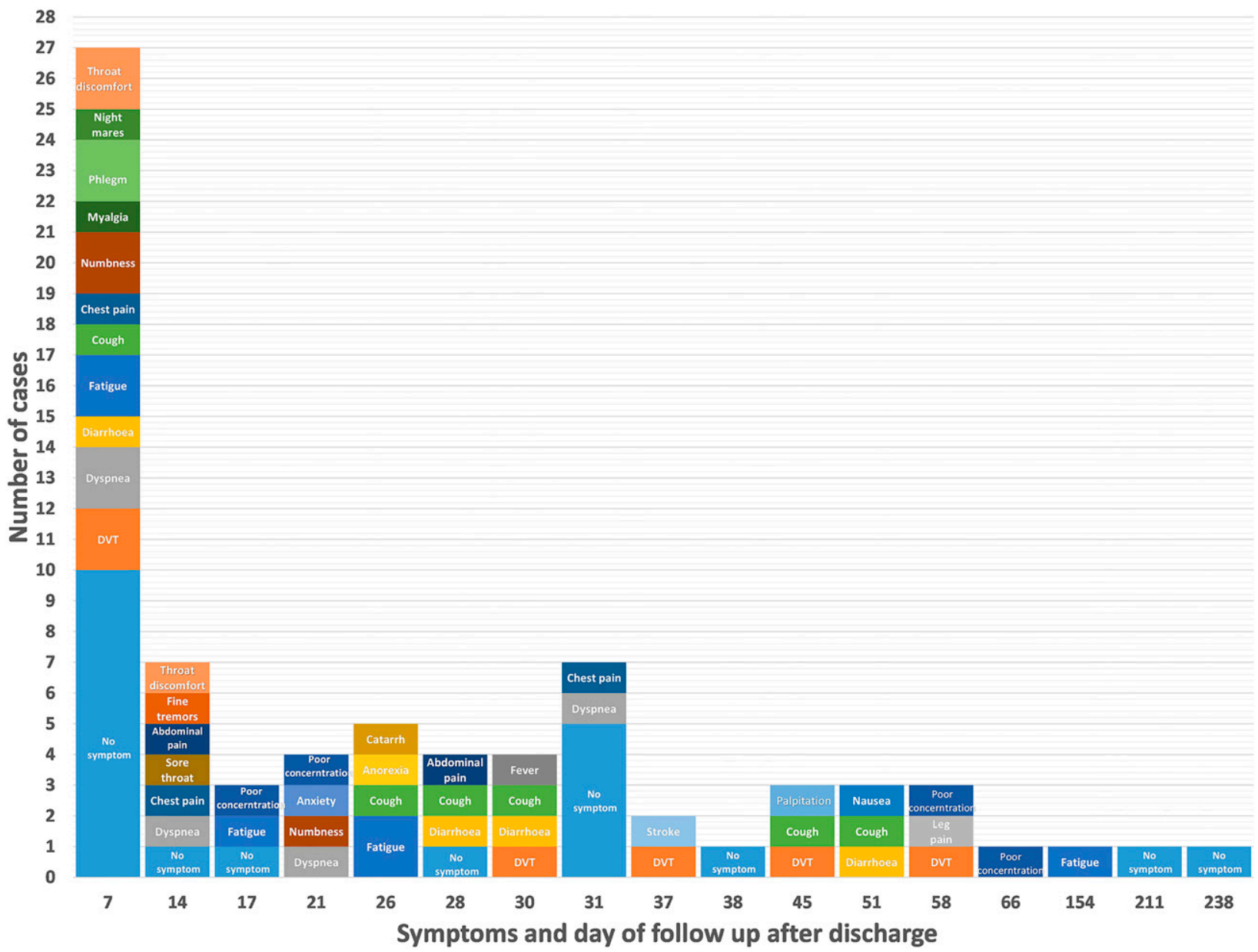

FIGURE 2. Clinical symptoms and signs of hospitalized coronavirus disease 2019 survivors in relation to number of days of follow-up after discharge. The follow-up period for study participants ranged from 7 to 238 days post-discharge. The majority of participants were asymptomatic on day 30 of follow-up. DVT = deep vein thrombosis. This figure appears in color at www.ajtmh.org.

confirmed to have deep vein thrombosis (DVT), which was corroborated by lower limb Doppler ultrasound (Figure 3). These cases of DVT resolved on days 14 and 238 of follow-up after use of compression stockings and oral anticoagulant therapy
(20 mg rivaroxaban twice daily). A 71-year-old who was diagnosed as hypertensive for the first time during hospitalization for COVID-19 complained of fatigue and excessive expectoration of mucus on day 7 of follow-up. On day 37 post-discharge,

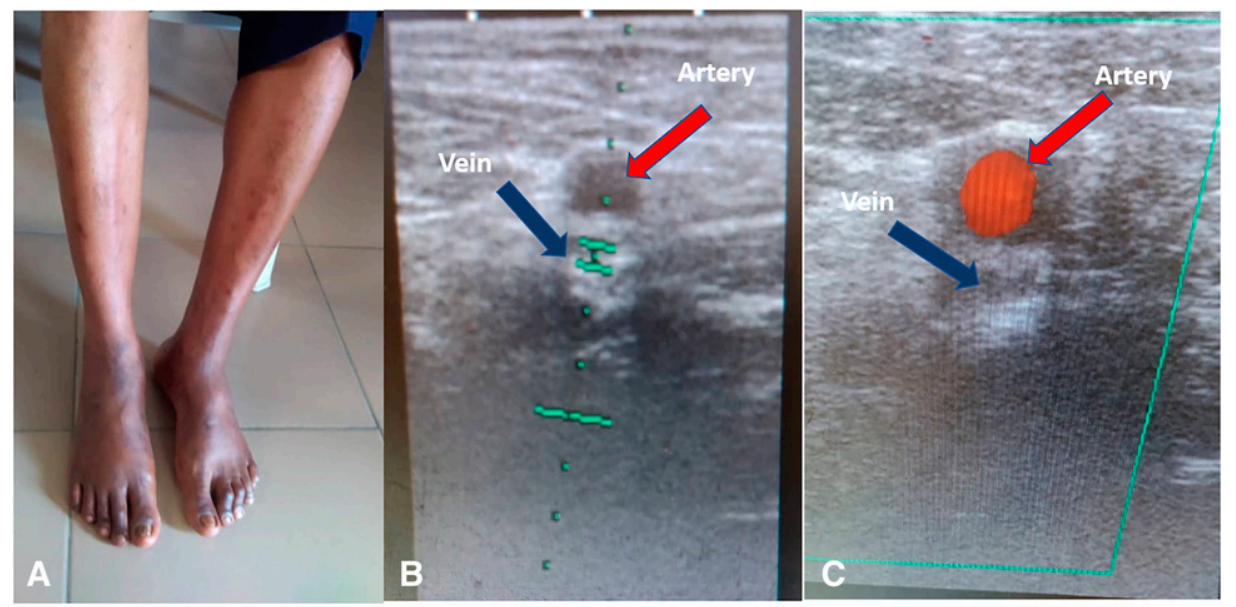

FIGURE 3. Deep vein thrombosis of lower limbs in a coronavirus disease 2019 survivor. (A) Both lower limbs are swollen, especially below both ankles. The right leg is slightly more swollen than the left. (B) Doppler ultrasound shows no evidence of calcification or atheroma in the right femoral artery. The right femoral vein is not compressible with evidence of thrombus within. (C) Color Doppler ultrasound shows evidence of blood flow in the right femoral artery and absence of blood flow in the right femoral vein. This figure appears in color at www.ajtmh.org. 
TABLE 1

Demographic and clinical characteristics of study participants in relation to development of post-acute coronavirus disease 2019 syndrome

\begin{tabular}{|c|c|c|c|c|}
\hline \multirow[b]{2}{*}{ Variable } & \multirow[b]{2}{*}{ Total } & \multicolumn{2}{|c|}{ Post-acute COVID-19 syndrome } & \multirow[b]{2}{*}{$P$ value } \\
\hline & & Yes & No & \\
\hline \multicolumn{5}{|l|}{ Gender, $n(\%)$} \\
\hline Male & $20(66.7)$ & $13(65)$ & $7(35)$ & 0.25 \\
\hline Female & $10(33.3)$ & $4(40)$ & $6(60)$ & - \\
\hline Age, y; median (IQR) & $46(33-52)$ & $49(34-52)$ & $45(21-48)$ & 0.17 \\
\hline \multicolumn{5}{|l|}{ Age group, y; $n(\%)$} \\
\hline$<18$ & $2(6.7)$ & $0(0)$ & $2(100)$ & 0.33 \\
\hline $18-59$ & $22(73.3)$ & $13(59.1)$ & 9 (40.9) & - \\
\hline$>59$ & $6(20)$ & $4(66.7)$ & 2 (33.3) & - \\
\hline Baseline $\mathrm{SpO}_{2}, \%$; median (IQR) & $95.5(91-98)$ & $95(90-96)$ & 96 (95-99) & $0.043^{*}$ \\
\hline \multicolumn{5}{|l|}{$\mathrm{SpO}_{2}$ category, $n(\%)$} \\
\hline $\mathrm{SpO}_{2} \geq 90 \%$ & $25(83.3)$ & $13(52)$ & $12(48)$ & 0.36 \\
\hline $\mathrm{SpO}_{2}<90 \%$ & 5 (16.7) & $4(80)$ & $1(20)$ & - \\
\hline \multicolumn{5}{|l|}{ Disease severity, $† n(\%)$} \\
\hline Mild & $14(46.7)$ & $5(35.7)$ & $9(64.3)$ & $0.029^{*}$ \\
\hline Moderate & 10 (33.3) & $6(60)$ & $4(40)$ & - \\
\hline Severe & $6(20)$ & $6(100)$ & $0(0)$ & - \\
\hline \multicolumn{5}{|l|}{ History of diabetes, $n(\%)$} \\
\hline Yes & $6(20)$ & $4(66.7)$ & 2 (33.3) & 0.672 \\
\hline No & $24(80)$ & $13(54.2)$ & $11(45.8)$ & - \\
\hline \multicolumn{5}{|l|}{ History of hypertension, $n(\%)$} \\
\hline Yes & $12(40)$ & $8(66.7)$ & $4(33.3)$ & 0.47 \\
\hline No & $18(60)$ & $9(50)$ & $9(50)$ & - \\
\hline Hospital stay, d; median (IQR) & $11(8-15)$ & $12(8-14)$ & $10(8-15)$ & 0.092 \\
\hline All participants, N (\%) & $30(100)$ & 17 (56.7) & $13(43.3)$ & - \\
\hline
\end{tabular}

he developed progressive right-side limb weakness associated with slurring of speech and worsening fatigue. A brain computed tomographic scan was normal. A diagnosis of ischemic stroke was made. After physiotherapy, anti-platelet therapy, and blood pressure control, limb weakness and slurred speech resolved completely within 2 weeks. At day 154 post-discharge, this patient complained of persistent fatigue but was otherwise clinically stable. A 45-year-old health-care worker complained of nighttime shortness of breath and nightmares on day 7 of follow-up. Nighttime shortness of breath persisted on day 21 of follow-up and was associated with dry cough. On clinical evaluation, vital signs and chest examination were essentially normal. Oxygen saturation by pulse oximetry remained $>98 \%$ at every follow-up visit. All symptoms resolved on day 31 of follow-up and the patient has remained asymptomatic at day 211 of follow-up.

Of the 17 cases of PCS, three (17.6\%) had persistent symptoms for more than 12 weeks (including fatigue, poor concentration, cough, and features of DVT), and 14 $(82.4 \%)$ had persisting symptoms for 3 to 12 weeks. Patients admitted with severe COVID-19 were more likely to develop PCS than those admitted with mild and moderate COVID-19 (Table 1).

\section{DISCUSSION}

Approximately $57 \%$ of our patients had PCS, with symptoms reported in almost every organ system. Approximately three symptoms were reported per patient over a follow-up period of 7 to 238 days. The development of PCS was associated significantly with baseline severity of COVID-19.

Our report suggest that $\mathrm{PCS}$ is common among hospitalized patients in our setting. This assertion is corroborated by a similar study from Lagos, Nigeria, where $40.6 \%$ of hospitalized adults reported symptoms suggestive of PCS. ${ }^{10}$ Studies from Europe, the United Kingdom, the United States, and Asia have reported PCS among $32 \%$ to $96 \%$ of hospitalized COVID-19 patients. $^{12-15}$

Cough, fatigue, and dyspnea were the most common symptoms reported in our cohort. This compares with most other studies across the globe. In addition to respiratory symptoms, we also reported symptoms in other organ systems, including gastrointestinal and hematological, and neuropsychiatric symptoms as well. Throat discomfort and phlegm were observed in some of our patients, but these symptoms have been reported rarely in previous literature. Two patients were observed to have clinical and radiological evidence of DVT requiring compression stockings and long-term anticoagulation before complete recovery. Postdischarge venous thromboembolism after hospital admission with COVID-19 has been reported by some studies from Europe ${ }^{16}$ and the United States, ${ }^{17}$ but the benefits of thromboprophylaxis in preventing venous thromboembolism among discharged COVID-19 patients remains uncertain. It is noteworthy that one of our patients, recently diagnosed as hypertensive, developed an ischemia stroke 1 month after discharge. It is plausible that, in addition to a history of hypertension, the increased procoagulant activity associated with COVID- $19^{2}$ contributed to the development of stroke in this patient. In other words, COVID-19 might have increased the risk of stroke in this patient. This agrees with previous studies in which COVID-19 was identified as an independent risk factor for stroke. ${ }^{18,19}$

The varied multisystemic manifestations of our patients may be related to the wide distribution of the preferred SARS-CoV-2 host receptor, angiotensin-converting enzyme 
2 in different tissues, ${ }^{20}$ and may be underlined by pathophysiological mechanisms associated with COVID-19, including direct viral toxicity, endothelial dysfunction, immune dysregulation, cytokine storm, and hypercoagulability with resultant thromobosis. ${ }^{2,21}$

Our results also suggest that some symptoms of PCS may persist for up to 3 to 9 months after discharge, and that initially asymptomatic COVID-19 survivors may later develop symptoms of PCS during follow-up. This finding calls for vigilance among clinicians caring for COVID-19 patients and emphasizes the need for long-term follow-up of COVID-19 patients even if they were asymptomatic at initial follow-up.

The development of PCS was not related to most clinical and demographic variables in our study. However, we found a significant relationship between baseline COVID-19 severity and PCS. Although there are some differences in study design, the increased risk of PCS in relation to increasing severity of COVID-19 is supported by a similar study from Lagos, Nigeria, ${ }^{10}$ and by studies from China ${ }^{12}$ and the United Kingdom. ${ }^{22}$ Conversely, studies from Spain ${ }^{15}$ and France ${ }^{23}$ did not demonstrate any relationship between baseline severity of COVID-19 and PCS.

COVID-19 reinfection is thought to be rare, ${ }^{24,25}$ and to our knowledge no case of reinfection has been published in the literature from Nigeria. One of our patients developed typical COVID-19 symptoms and had a positive PCR test about 4 weeks after a negative PCR test and apparent complete recovery from the first episode. A COVID-19 reinfection or relapse is plausible in this patient. Unfortunately, because viral culture was not done to determine viability of viral particles, and genomic sequencing was not available to identify similarities in infecting viral strains, we could not confirm this assertion. Further studies are needed to define the scope of repeat COVID-19 infection in Nigeria.

Our study has some limitations. First, the sample size was small, and the limited study participants precluded multivariate analysis of independent predictors of PCS. In view of the sample size, our study findings may not be generalizable to other hospitalized COVID-19 survivors in Nigeria. However, it is our view that our findings are reflective of the Nigeria situation because they are largely comparable to another Nigeria study in which 274 patients were enrolled. ${ }^{10}$ Second, we could not conduct additional investigations such as chest imaging, spirometry, D-dimer tests, and exercise tolerance tests, among others, to characterize further the pathological features of organ dysfunction among our patients. Third, our results were based on retrospective review of hospital records and, as such, it is possible that patients who did not present for follow-up developed symptoms that were not documented by our study. There is also a possibility of recall bias as some of our patients presenting for follow-up could have forgotten or neglected to mention some symptoms they experienced. A future prospective study is required to characterize fully the prevalence and determinants of PCS in Nigeria.

In conclusion, PCS is common in our setting, characterized by short- and long-term multisystemic symptoms-mostly cough, fatigue, and dyspnea. The development of PCS was associated significantly with baseline COVID-19 severity. Future multicenter long-term prospective studies are needed to characterize fully the prevalence and determinants of PCS among COVID-19 survivors in Nigeria.
Received May 6, 2021. Accepted for publication June 14, 2021.

Published online July 16, 2021.

Acknowledgment: The American Society of Tropical Medicine and Hygiene has waived the Open Access fee for this article due to the ongoing COVID-19 pandemic and has assisted with publication expenses.

Authors' addresses: Dimie Ogoina and Hendris Izibewule James, Department of Internal Medicine, Niger Delta University Teaching Hospital, Okolobiri, Bayelsa State, Nigeria, E-mails: dimieogoina@gmail. com and drhendrisjames@gmail.com. Stanley Zikeyi Ogoinja, Department of Radiology, Niger Delta University Teaching Hospital, Okolobiri, Bayelsa State, Nigeria, E-mail: dimieogoina@gmail.com.

This is an open-access article distributed under the terms of the Creative Commons Attribution (CC-BY) License, which permits unrestricted use, distribution, and reproduction in any medium, provided the original author and source are credited.

\section{REFERENCES}

1. World Health Organization, 2020. Clinical Management of Severe Acute Respiratory Infection When COVID-19 is Suspected. Geneva, Switzerland: WHO, 1-82. Available at: https://www. who.int/publications-detail/clinical-management-of-severeacute-respiratory-infection-when-novel-coronavirus-(ncov)infection-is-suspected. Accessed May 23, 2020.

2. Nalbandian A et al., 2021. Post-acute COVID-19 syndrome. Nat Med 27: 601-615.

3. Amenta EM, Spallone A, Rodriguez-Barradas MC, El Sahly HM, Atmar RL, Kulkarni PA, 2020. Postacute COVID-19: an overview and approach to classification. Open Forum Infect Dis 7: 1-7.

4. Kaiter L, 2021. Fauci Introduces New Acronym for Long COVID. Available at: https://www.medscape.com/viewarticle/946419. Accessed May 2, 2021.

5. Logue JK, Franko NM, McCulloch DJ, McDonald D, Magedson A, Wolf CR, Chu HY, 2021. Sequelae in adults at 6 months after COVID-19 infection. JAMA Netw Open 4: 1-9.

6. Nigeria Centre for Disease Control, 2021. COVID-19 Nigeria. Available at: https://covid19.ncdc.gov.ng/. Accessed April 25, 2021.

7. Abayomi A et al., 2021. Presenting symptoms and predictors of poor outcomes among 2,184 patients with COVID-19 in Lagos State, Nigeria. Int J Infect Dis 102: 226-232.

8. Elimian KO et al., 2020. Patient characteristics associated with COVID-19 positivity and fatality in Nigeria: retrospective cohort study. BMJ Open 10: 44079.

9. Elimian KO et al., 2020. Descriptive epidemiology of coronavirus disease 2019 in Nigeria, 27 February-6 June 2020. Epidemiol Infect 148: e208.

10. Osikomaiya B et al., 2021. "Long COVID": persistent COVID-19 symptoms in survivors managed in Lagos State, Nigeria. BMC Infect Dis 21: 304.

11. Nigeria Centre of Disease Control, 2020. National Interim Guidelines for Clinical Management of COVID-19. Available at: https://www.ncdc.gov.ng/themes/common/docs/protocols/ 177_1584210847.pdf. Accessed April 25, 2021.

12. Huang $C$ et al., 2021. 6-Month consequences of COVID-19 in patients discharged from hospital: a cohort study. Lancet 397: 220-232.

13. Weerahandi $H$ et al., 2021. Post-discharge health status and symptoms in patients with severe COVID-19. J Gen Intern Med 36: 738-745.

14. Carfi A, Bernabei R, Landi F, 2020. Persistent symptoms in patients after acute COVID-19. JAMA 324: 603-605.

15. Moreno-Pérez O et al., 2021. Post-acute COVID-19 syndrome: incidence and risk factors: a Mediterranean cohort study. $J$ Infect 82: 378-383.

16. Roberts LN, Whyte MB, Georgiou L, Giron G, Czuprynska J, Rea C, Vadher B, Patel RK, Gee E, Arya R, 2020. Postdischarge venous thromboembolism following hospital admission with COVID-19. Blood 136: 1347-1350. 
17. Patell R, Bogue T, Koshy A, Bindal $P$, Merrill M, Aird WC, Bauer KA, Zwicker JI, 2020. Postdischarge thrombosis and hemorrhage in patients with COVID-19. Blood 136: 1342-1346.

18. Belani $P$ et al., 2020. COVID-19 is an independent risk factor for acute ischemic stroke. AJNR Am J Neuroradiol 41: 1361-1364.

19. Zakeri A, Jadhav AP, Sullenger BA, Nimjee SM, 2021. Ischemic stroke in COVID-19-positive patients: an overview of SARSCoV-2 and thrombotic mechanisms for the neurointerventionalist. J Neurointerv Surg 13: 202-206.

20. Datta PK, Liu F, Fischer T, Rappaport J, Qin X, 2020. SARS-CoV-2 pandemic and research gaps: understanding SARS-CoV-2 interaction with the ACE2 receptor and implications for therapy. Theranostics 10: 7448-7464.

21. Alyammahi SK, Abdin SM, Alhamad DW, Elgendy SM, Altell AT, Omar HA, 2021. The dynamic association between COVID-19 and chronic disorders: an updated insight into prevalence, mechanisms and therapeutic modalities. Infect Genet Evol 87: 104647.

22. Halpin SJ et al., 2021. Postdischarge symptoms and rehabilitation needs in survivors of COVID-19 infection: a cross-sectional evaluation. J Med Virol 93: 1013-1022.

23. Garrigues E et al., 2020. Post-discharge persistent symptoms and health-related quality of life after hospitalization for COVID-19. J Infect 81: e4-e6.

24. Ledford $\mathrm{H}, 2021$. COVID reinfections are unusual, but could still help the virus to spread. Nature. Available at: https://www. nature.com/articles/d41586-021-00071-6. Accessed April 25, 2021.

25. Hansen CH, Michlmayr D, Gubbels SM, Mølbak K, Ethelberg S, 2021. Assessment of protection against reinfection with SARS-CoV-2 among 4 million PCR-tested individuals in Denmark in 2020: a population-level observational study. Lancet 397: 1204-1212. 